\title{
WAVEGUIDING IN THIN FILMS WITH QUADRATIC MAGNETO-OPTICAL MEDIUM
}

\author{
J. Pištora ${ }^{a, *}$, A. Domański ${ }^{b}$, O. BÁrta ${ }^{a}, \mathrm{~F} \cdot \mathrm{StanťK}^{a}, \mathrm{~K} \cdot \operatorname{Postava}^{a}$, \\ D. CIPRIAN ${ }^{a}$ AND I. KOPŘIVA ${ }^{a}$ \\ ${ }^{a}$ Department of Physics, Technical University Ostrava. \\ 17. listopadu 15, 70833 Ostrava, Czech Republic \\ ${ }^{b}$ Faculty of Physics, Warsaw University of Technology \\ Koszykowa 75, 00-662 Warsaw, Poland
}

\begin{abstract}
The waveguiding in thin planar structures with quadratic magneto-optical medium is analysed. The permittivity tensor elements with quadratic magneto-optical constants are specified. From synchronous condition for guided modes in layered system with magnetic ordering the dispersion relation of waveguide is presented. The reflection coefficients for $s$-polarized light at transversal, longitudinal, and polar configurations as incidence angle function are described. The influence of quadratic magneto-optical effect on the resonant character of the waveguiding term is described in detail. The theory covers the situation in which the optical response of the structure is linear in the amplitude of the incident wave.
\end{abstract}

PACS numbers: 78.20.Ls

\section{Introduction}

Magneto-optical reflection measurements and eigenmode polarization states are influenced not only by linear magneto-optical (MO) effects in magnetization, but also by quadratic ones (second-order effects). The quadratic MO effects in reflection have been observed at ferromagnetic layers with in-plane magnetization $[1,2]$. The quadratic contribution resulting from the product of the longitudinal and the transversal magnetization components affects the correct hysteresis loop measurement by the longitudinal Kerr effect [3]. The propagation of the light in the planar structure with quadratic effects is based on calculation of the eigenmodes in a magneto-optical medium with help of $4 \times 4$ Yeh's matrix formalism [4].

* corresponding author; e-mail: jaromir.pistora@vsb.cz 


\section{Permittivity tensor and eigenmode polarization}

Generally one can write for the relative permittivity tensor $\varepsilon_{\mathrm{r}}$

$$
\varepsilon_{\mathrm{r}}=\left(\begin{array}{lll}
\varepsilon_{x x} & \varepsilon_{x y} & \varepsilon_{x z} \\
\varepsilon_{y x} & \varepsilon_{y y} & \varepsilon_{y z} \\
\varepsilon_{z x} & \varepsilon_{z y} & \varepsilon_{z z}
\end{array}\right) .
$$

The elements of $\varepsilon_{i j}$ can be specified as $[5,6]$

$$
\begin{aligned}
& \varepsilon_{x x}=\varepsilon_{0}\left\{1+\left[\left(f_{11}-f_{12}\right) Q_{\mathrm{T}}^{2}+f_{12}\left(Q_{\mathrm{P}}^{2}+Q_{\mathrm{L}}^{2}+Q_{\mathrm{T}}^{2}\right)\right]\right\}, \\
& \varepsilon_{x y}=\varepsilon_{0}\left(\mathrm{i} Q_{\mathrm{P}}+2 f_{44} Q_{\mathrm{L}} Q_{\mathrm{T}}\right), \\
& \varepsilon_{x z}=\varepsilon_{0}\left(-\mathrm{i} Q_{\mathrm{L}}+2 f_{44} Q_{\mathrm{P}} Q_{\mathrm{T}}\right), \\
& \varepsilon_{y x}=\varepsilon_{0}\left(-\mathrm{i} Q_{\mathrm{P}}+2 f_{44} Q_{\mathrm{L}} Q_{\mathrm{T}}\right), \\
& \varepsilon_{y y}=\varepsilon_{0}\left\{1+\left[\left(f_{11}-f_{12}\right) Q_{\mathrm{L}}^{2}+f_{12}\left(Q_{\mathrm{P}}^{2}+Q_{\mathrm{L}}^{2}+Q_{\mathrm{T}}^{2}\right)\right]\right\}, \\
& \varepsilon_{y z}=\varepsilon_{0}\left(\mathrm{i} Q_{\mathrm{T}}+2 f_{44} Q_{\mathrm{L}} Q_{\mathrm{P}}\right), \\
& \varepsilon_{z x}=\varepsilon_{0}\left(\mathrm{i} Q_{\mathrm{L}}+2 f_{44} Q_{\mathrm{P}} Q_{\mathrm{T}}\right), \\
& \varepsilon_{z y}=\varepsilon_{0}\left(-\mathrm{i} Q_{\mathrm{T}}+2 f_{44} Q_{\mathrm{L}} Q_{\mathrm{P}}\right), \\
& \varepsilon_{z z}=\varepsilon_{0}\left\{1+\left[\left(f_{11}-f_{12}\right) Q_{\mathrm{P}}^{2}+f_{12}\left(Q_{\mathrm{P}}^{2}+Q_{\mathrm{L}}^{2}+Q_{\mathrm{T}}^{2}\right)\right]\right\},
\end{aligned}
$$

where $f_{11}, f_{12}$, and $f_{44}$ are quadratic MO constants, $Q_{\mathrm{L}}, Q_{\mathrm{T}}$, and $Q_{\mathrm{P}}$ are linear magneto-optical parameters proportional to the longitudinal, transversal, and polar magnetization components, respectively. The symbol $\varepsilon_{0}$ specifies the non-magnetic permittivity. The tensor elements (2) correspond to the case when the cubic crystal axes are parallel to the coordinate axes.

Assuming the plane monochromatic wave solution,

$$
\boldsymbol{E}=\boldsymbol{E}_{0} \exp [\mathrm{i}(\omega t-\boldsymbol{k})] \text {. }
$$

The coordinate system can always be chosen in such a way that the radiation wave vector in thin film is restricted to the $y z$-plane (see Fig. 1)

$$
\boldsymbol{k}=k_{y} \hat{\boldsymbol{y}}+k_{z} \hat{z}=k_{\mathrm{v}}\left(N_{y} \hat{\boldsymbol{y}}+N_{z} \hat{z}\right)
$$

where $\hat{y}$ and $z$ are the unit vectors, $k_{\mathrm{v}}=\omega / c=2 \pi / \lambda$ is a wave number in vacuum.

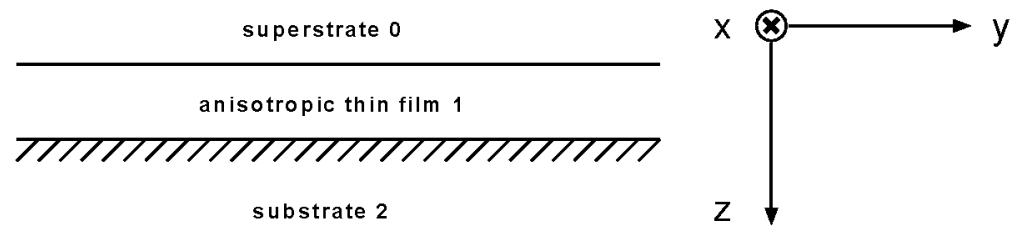

Fig. 1. Geometrical configuration.

By application of Yeh's $4 \times 4$ matrix formalism to the MO multilayers [7, 8] one can specify the eigenmode polarizations in each anisotropic thin film for transversal, polar, and longitudinal configuration [9]. The relation between the 
field in the $(n-1)$-th layer at the interface $z=z^{(n-1)}$ and the field in the $n$-th layer at interface $z=z^{(n)}$ is obtained by the transfer matrix

$$
\boldsymbol{T}^{(n-1, n)}=\left(\boldsymbol{D}^{(n-1)}\right)^{-1} \boldsymbol{D}^{(n)} \boldsymbol{P}^{(n)},
$$

where $\boldsymbol{D}$ is the dynamic matrix and $\boldsymbol{P}$ is the propagation matrix, which is diagonal. The product of transfer matrices specifies the total matrix $M$ of the structure

$$
\boldsymbol{M}=\left(\begin{array}{llll}
M_{11} & M_{12} & M_{13} & M_{14} \\
M_{21} & M_{22} & M_{23} & M_{24} \\
M_{31} & M_{32} & M_{33} & M_{34} \\
M_{41} & M_{42} & M_{43} & M_{44}
\end{array}\right) .
$$

As shown by Yeh [4] the condition

$$
M_{11} M_{33}-M_{13} M_{31}=0
$$

presents the dispersion relation of the waveguide formed by the structure.

\section{Waveguiding in anisotropic planar structure with magnetic ordering}

From synchronous condition for waveguiding in planar system (7) we can derive the dispersion relation of waveguide with magnetic ordering

$$
\begin{aligned}
& 1-r_{++}^{(10)} r_{++}^{(12)} P_{1} P_{2}-r_{--}^{(10)} r_{--}^{(12)} P_{3} P_{4}-r_{+-}^{(10)} r_{-+}^{(12)} P_{2} P_{3}-r_{-+}^{(10)} r_{+-}^{(12)} P_{1} P_{4} \\
& +\left(r_{++}^{(10)} r_{--}^{(10)}-r_{+-}^{(10)} r_{-+}^{(10)}\right)\left(r_{++}^{(12)} r_{--}^{(12)}-r_{-+}^{(12)} r_{+-}^{(12)}\right) P_{1} P_{2} P_{3} P_{4}=0 .
\end{aligned}
$$

The reflection coefficients $r^{(i j)}$ are, in general, complex; the signs + and - correspond to the right- and left-handed circular polarization. The elements $P_{l=1,2,3,4}$ specify the light propagation in anisotropic layer

$$
\begin{array}{ll}
P_{1}=\exp \left(-\mathrm{i} k_{\mathrm{v}} N_{z 1} d\right), & P_{2}=\exp \left(i k_{\mathrm{v}} N_{z 2} d\right), \\
P_{3}=\exp \left(-\mathrm{i} k_{\mathrm{v}} N_{z 3} d\right), & P_{4}=\exp \left(\mathrm{i} k_{\mathrm{v}} N_{z 4} d\right) .
\end{array}
$$

From Eq. (8) we can see that the guided modes in magneto-optical waveguide are elliptically polarized waves. The reflection coefficients from circular base can be simply transformed to the amplitude reflection coefficients in $x y z$-base [10]

$$
\begin{aligned}
& r_{--}=\frac{1}{2}\left[r_{s s}+r_{p p}-\mathrm{i}\left(r_{p s}-r_{s p}\right)\right], \\
& r_{++}=\frac{1}{2}\left[r_{s s}+r_{p p}+\mathrm{i}\left(r_{p s}-r_{s p}\right)\right], \\
& r_{+-}=\frac{1}{2}\left[r_{s s}-r_{p p}+\mathrm{i}\left(r_{p s}+r_{s p}\right)\right], \\
& r_{-+}=\frac{1}{2}\left[r_{s s}-r_{p p}-\mathrm{i}\left(r_{p s}+r_{s p}\right)\right] .
\end{aligned}
$$

The $s$ polarization is perpendicular to the plane of incidence (along $x$-axis), $p$ polarization lies in the plane of incidence ( $y z$-plane). For example, the $r_{p s}$ reflection coefficient is defined as the ratio of the $x(s)$ component of the reflected light to the $y(p)$ component of the incident one. 
Magneto-optical parameters $Q_{\mathrm{P}}, Q_{\mathrm{L}}$, and $Q_{\mathrm{T}}$ are proportional to the polar $M_{\mathrm{P}}$, longitudinal $M_{\mathrm{L}}$, and transversal $M_{\mathrm{T}}$ magnetization components [6]

$$
Q_{\mathrm{P}}=Q \frac{M_{\mathrm{P}}}{M_{\mathrm{S}}}, \quad Q_{\mathrm{L}}=Q \frac{M_{\mathrm{L}}}{M_{\mathrm{S}}}, \quad Q_{\mathrm{T}}=Q \frac{M_{\mathrm{T}}}{M_{\mathrm{S}}}
$$

where $M_{\mathrm{S}}$ is the magnetization in saturation and $Q$ is the linear magneto-optical parameter defined by Voigt. The dispersion relation of guided modes in the layer with magnetic ordering (8) depends on the reflection coefficients related to the boundaries thin film-superstrate and thin film-substrate, and on the propagation factors $P_{l}$. General analytic formulae for reflection coefficients for thin film structure with linear and quadratic magnetic ordering are too extensive. For a cubic crystal the magnetization effects on the reflection coefficients are summarized in Table I. In the linear approximation (without quadratic magneto-optical effect) at transversal geometry the guided modes are separated. We can study the influence of $M_{\mathrm{T}}$ on waveguiding for $p$-polarized waves. Any mode conversion is observed in this case. As was demonstrated in Table I, the reflection coefficients $r_{s s}$ and $r_{p p}$ up to quadratic approximation are not sensitive to the mutual polar and longitudinal term $M_{\mathrm{P}}, M_{\mathrm{L}}$.

TABLE I

Reflection coefficient dependencies on the magnetization components.

\begin{tabular}{c|c|c|c}
\hline \hline & Non-magnetic & Linear & Quadratic \\
\hline$r_{s s}$ & Fresnel formula & - & $M_{\mathrm{P}}^{2}, M_{\mathrm{L}}^{2}, M_{\mathrm{T}}^{2}, M_{\mathrm{L}} M_{\mathrm{T}}$ \\
\hline$r_{p p}$ & Fresnel formula & $M_{\mathrm{T}}$ & $M_{\mathrm{P}}^{2}, M_{\mathrm{L}}^{2}, M_{\mathrm{T}}^{2}, M_{\mathrm{L}} M_{\mathrm{T}}$ \\
\hline$r_{s p}, r_{p s}$ & - & $M_{\mathrm{P}}, M_{\mathrm{L}}$ & $M_{\mathrm{L}}^{2}, M_{\mathrm{T}}^{2}, M_{\mathrm{P}} M_{\mathrm{T}}, M_{\mathrm{L}} M_{\mathrm{T}}$
\end{tabular}

The process of light waveguiding can be generally divided into two basic parts - 1st is the reflection on the thin film-substrate boundary and on the thin film-superstrate one, 2nd contribution is connected with propagation of the light beam in magneto-optical layer. The numerical solution of the waveguiding equation (8) for the transversal ordering (parameters: $n^{(0)}=1.0, N^{(1)}=2.87-$ $0.07 \mathrm{i}, Q_{\varepsilon}^{(1)}=0.0386-0.0034 \mathrm{i}, f_{\varepsilon}^{(1)}=0.540-0.568 \mathrm{i}, d^{(1)}=1 \mu \mathrm{m}, n^{(2)}=1.735, \lambda=$ $632.8 \mathrm{~nm}$ ) is presented in Figs. 2-5. Figure 2 describes waveguiding at transversal geometry for $s$-polarized light without quadratic effect (full line), the dashed line demonstrates the quadratic element contribution. The most important factor for the generation of guided modes is level of the absorption in thin film. To observe the guided mode modulation by quadratic MO element, the imaginary part of the thin film refractive index has been chosen as zero or 0.07 in this case. The real value of the absorption $\left(\varepsilon_{0}^{(1)}=(2.87-3.17 \mathrm{i})^{2}\right)$ totally suppresses the resonant character of the synchronous relation (8). 

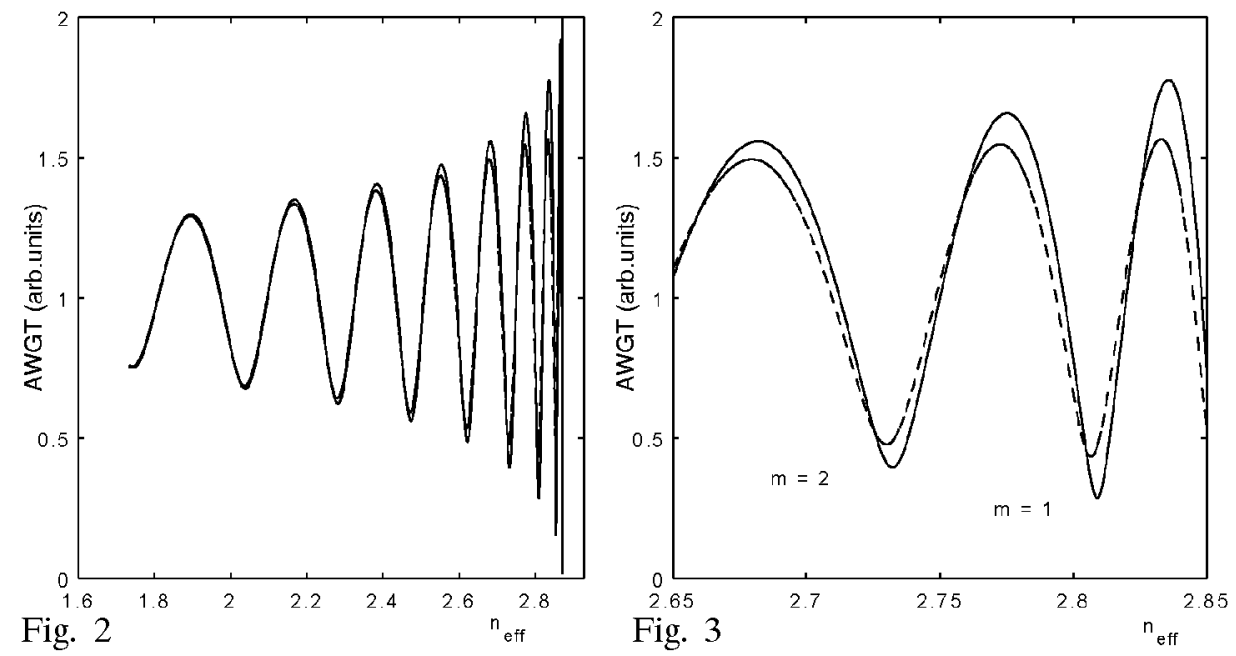

Fig. 2. The absolute value of the dispersion relation of waveguide (8) for $s$-polarized light (dashed line includes the quadratic MO effect) plotted as a function of effective refractive index $n_{\text {eff }}$ in thin film.

Fig. 3. The absolute value of the dispersion relation of waveguide (8) for $s$-polarized light (dashed line includes the quadratic MO effect) plotted as a function of effective refractive index $n_{\text {eff }}$ in thin film - detailed situation for 1 st and 2 nd mode order.

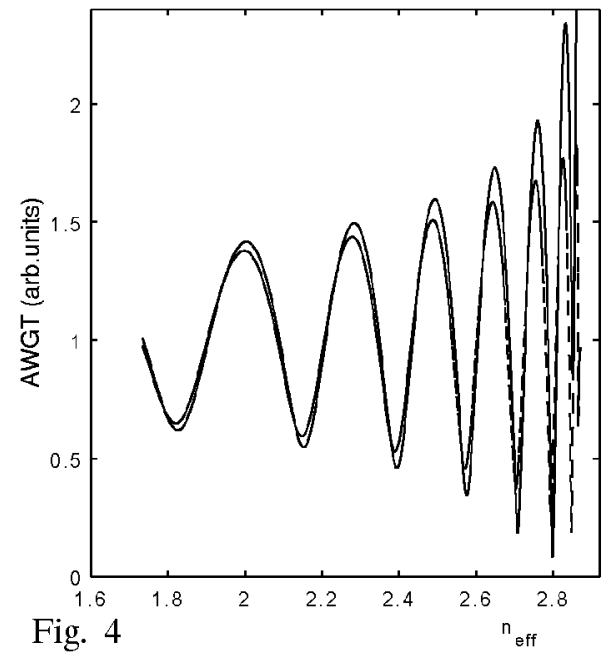

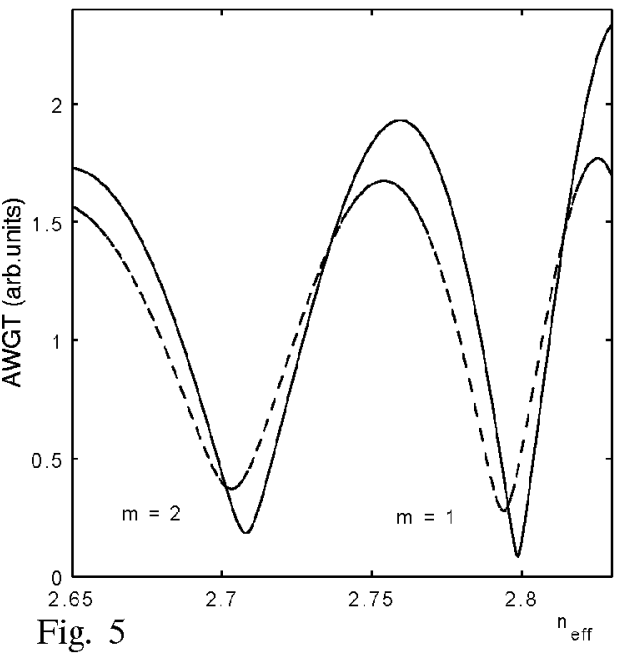

Fig. 5

Fig. 4. The absolute value of the dispersion relation of waveguide ( 8 ) for $p$-polarized light (dashed line includes the quadratic MO effect) plotted as a function of effective refractive index $n_{\text {eff }}$ in thin film.

Fig. 5. The absolute value of the dispersion relation of waveguide (8) for $p$-polarized light (dashed line includes the quadratic MO effect) plotted as a function of effective refractive index $n_{\text {eff }}$ in thin film - detailed situation for 1st and 2nd mode order. 


\section{Conclusions}

In comparison of the curves presented in Figs. 2, 4 (transversal geometry) one can observe the important minima position changes (see Table II). These position shifts are generated generally by the following contributions:

- the absorption level in thin film,

- the linear magneto-optical effect,

- the quadratic magneto-optical effect.

TABLE II

Effective indices of guided modes and changes generated by quadratic magneto-optical effect (2nd and 3rd column for s-polarized light without and with quadratic MO phenomena contribution; 4th and 5th column are the same for $p$ polarization; $m$ is the mode order).

\begin{tabular}{c|c|c|c|c}
\hline \hline \multirow{2}{*}{$m$} & \multicolumn{2}{|c|}{$n_{\mathrm{eff}_{m}}^{\mathrm{TE}}$} & \multicolumn{2}{|c}{$n_{\mathrm{eff}}^{\mathrm{TM}}$} \\
\cline { 2 - 5 } & linear & quadratic & linear & quadratic \\
\hline 0 & 2.86915 & 2.86903 & 2.85134 & 2.84705 \\
1 & 2.85422 & 2.85182 & 2.79866 & 2.79398 \\
2 & 2.80904 & 2.80670 & 2.70795 & 2.70320 \\
3 & 2.73230 & 2.73004 & 2.57552 & 2.57073 \\
4 & 2.62168 & 2.61953 & 2.39448 & 2.38962 \\
5 & 2.47345 & 2.47144 & 2.15262 & 2.14761 \\
6 & 2.28189 & 2.28006 & 1.82589 & 1.82057
\end{tabular}

The level of absorption transforms the resonant amplitude character of the waveguiding term. It means that for high level absorption one cannot realize waveguiding. From Table $\mathrm{I}$ is evident that $r_{s s}$ coefficients do not depend on linear magneto-optical effect at transversal configuration. The minima shifts for the same polarization raised by quadratic effect are from $10^{-4}$ to $2 \times 10^{-3}$ (as function of guided mode order). For $p$-polarized light we observe the discussed shifts in the frame of $4 \times 10^{-3}$. Because with the help of the dark mode spectroscopy the effective indices of refraction can be specified with accuracy of $10^{-3}$ (and better), the analysis of the guided modes in the planar structures with magnetic ordering can extend the experimental possibilities for the study of nonlinear magneto-optical phenomena.

\section{Acknowledgments}

This work has been partially supported by the Ministry of Education, Youth and Sport of the Czech Republic (grants KONTAKT No. ME 174 and ME 175) and by research project CEZ: J17/98:272400019. 


\section{References}

[1] K. Postava, H. Jaffres, A. Schuhl, F. Nguien Van Dau, M. Goiran, A.R. Fert, J. Magn. Magn. Mater. 172, 199 (1998).

[2] R.M. Osgood III, S.D. Bader, B.M. Clemens, R.L. White, H. Matsuyama, J. Magn. Magn. Mater. 182, 297 (1998).

[3] K. Postava, J. Pištora, R. Kantor, A.R. Fert, M. Goiran, A. Schuhl, F. Nguien Van Dau, Proc. SPIE 3320, 264 (1998).

[4] P. Yeh, Surf. Sci. 96, 41 (1980).

[5] I. Kopřiva, D. Hrabovský, K. Postava, D. Ciprian, J. Pištora, Proc. SPIE 4016, $54(2000)$.

[6] K. Postava, J. Pištora, D. Ciprian, D. Hrabovský, M. Lesñák, A.R. Fert, Proc. SPIE 3820, 412 (1999).

[7] J. Pištora, J. Vlček, O. Bárta, K. Postava, I. Kopřiva, Jemná mechanika a optika $45,235(2000)$.

[8] Š. Višñovský, Czech. J. Phys. B 36, 625 (1986).

[9] J. Pištora, O. Bárta, J. Vlček, D. Ciprian, D. Hrabovský, I. Vávra, V. Smatko, E. Kováčová, K. Postava, I. Kopřiva, J. Opt. Soc. Am. A, to be published.

[10] K. Postava, Ph.D. thesis, 1997. 